\title{
Liquid chromatograph-mass spectrometry-based plasma metabonomics study on the effects of moxibustion with seed-sized moxa cone on hyperlipidaemia
}

Qinghua Shao ${ }^{1,2 \#}$, Jie Cheng ${ }^{3 \#}$, Yi $\mathrm{Li}^{4}$ and Guangxia $\mathrm{Ni}^{3}$

${ }^{1}$ Affiliated Hospital of Integrated Traditional Chinese and Western Medicine, Nanjing University of Chinese Medicine, Nanjing, 210023, China

${ }^{2}$ Jiangsu Province Academy of Traditional Chinese Medicine, Nanjing, 210028, China

${ }^{3}$ The Second Clinical College, Nanjing University of Chinese Medicine, Nanjing, 210023, China

${ }^{4}$ Graduated School, Nanjing University of Chinese Medicine, Nanjing, 210023, China

\#The authors contribute equally to the study.

Corresponding author:

Guangxia Ni: The Second Clinical College, Nanjing University of Chinese Medicine, 138 Xianlin Road, Nanjing, 210023, China

E-mail: xgn66@163.com

Yi Li: Graduated School, Nanjing University of Chinese Medicine, 138 Xianlin Road, Nanjing, 210023, China

E-mail: liyi@njucm.edu.cn 


\begin{abstract}
:
Hyperlipidaemia (HLP) is a disorder with disturbed lipid metabolism and a major risk factor for various diseases. This study focused on the effect of moxibustion with seed-sized moxa cone on HLP and the relative metabolites and pathways. Levels of total cholesterol (TC), triglyceride (TG) and low-density lipoprotein cholesterol (LDC-C) in healthy controls (HC), HLP patients, HLP patients before (BMT) and after (AMT) moxibustion treatment were measured. Using the liquid chromatograph-mass spectrometry (LC-MS) technique, we detected the plasma metabolites in the aforementioned groups. Pathway analysis was performed for the differential metabolites. Results revealed significantly high levels of TC, TG and LDC-C and low levels of HDC-C were measured in HLP compared to HC. After moxibustion treatment, the levels of TC and TG significantly decreased. Total 87 and 51 differential metabolites were identified in HLP vs HC and AMT vs BMT, respectively, and 21 were common differential metabolites. The differential metabolites in HLP vs HC and AMT vs BMT both significantly enriched in the pathways of Glycerophospholipid metabolism and Sphingolipid metabolism. In conclusion, moxibustion with seed-sized moxa cone effectively improved HLP, might be by affecting the levels of $\mathrm{TC}$ and $\mathrm{TG}$, and the regulating of the Glycerophospholipid metabolismand Sphingolipid metabolism pathways.
\end{abstract}

Key words: hyperlipidaemia; metabolomics; phosphatidylcholine; sphingomyelin

\title{
1. Introduction
}

Hyperlipidaemia (HLP) is the disease with disordered lipid metabolism including high levels of total cholesterol (TC), triglyceride (TG) and low-density lipoprotein cholesterol (LDC-C) as along with a low level of high-density lipoprotein cholesterol (HDC-C) [1]. HLP increased the risk of many other diseases, such as cardiovascular diseases (CVD) [2], diabetes mellitus [3,4], periodontitis [3], coronary artery atherosclerosis [5], coronary heart disease [6] and sleep related-breathing disorders [7], becoming a heavy burden for human healthy. Thus, the development of effective therapies is meaningful and important.

Moxibustion and acupuncture had been considered as potential treatments for hyperlipidaemia [8-10]. Moxibustion with seed-sized moxa cone has been reported to protect heart function by inhibiting the apoptosis of myocardial cells in mice [11]. It can relieve myocardial ischemia effectively in ischemia-reperfusion injury rats [12]. Moxibustion with seed-sized moxa cone also had an effect of alleviating the pain caused by cervical spondylosis and delaying liver cancer precancerous lesion in rats $[13,14]$. However, the effect of moxibustion with seed-sized moxa cone on hyperlipidaemia and relative metabolic profile are still unknown.

In the present study, we performed the plasma metabolic profiles of HLP patients, healthy controls (HC) and HLP patients with moxibustion treatment using liquid 
chromatograph-mass spectrometry (LC-MS) technique. The alterations of metabolites in HLP and HLP patients with moxibustion treatment were observed, and the pathways that the differential metabolites enriched were predicted, respectively. This study might provide potential biomarkers and effective therapeutic strategy for hyperlipidaemia.

\section{Results}

\subsection{Clinical chemistry results}

The clinical data showed that, compared to the HC group, HLP patients had significantly higher levels of TG, TC and LDC-C and a markedly lower level of HDC-C (Fig. 1A). After treated by moxibustion with seed-sized moxa cone for 8 weeks, the concentrations of TC $(4.96 \pm 0.15 \mathrm{mmol} / \mathrm{L})$ and TG $(1.69 \pm 0.35 \mathrm{mmol} / \mathrm{L})$ were decreased significantly in the AMT group (Fig. 1B). Although there were no significant differences in the levels of LDC-C and HDC-C between AMT group and BMT group, the levels of LDC-C $(3.21 \pm 0.18 \mathrm{mmol} / \mathrm{L}<3.37 \mathrm{mmol} / \mathrm{L})$ and HDC-C $(1.23 \pm 0.07 \mathrm{mmol} / \mathrm{L}>1.04 \mathrm{mmol} / \mathrm{L})$ in the AMT group were both in the normal range. 

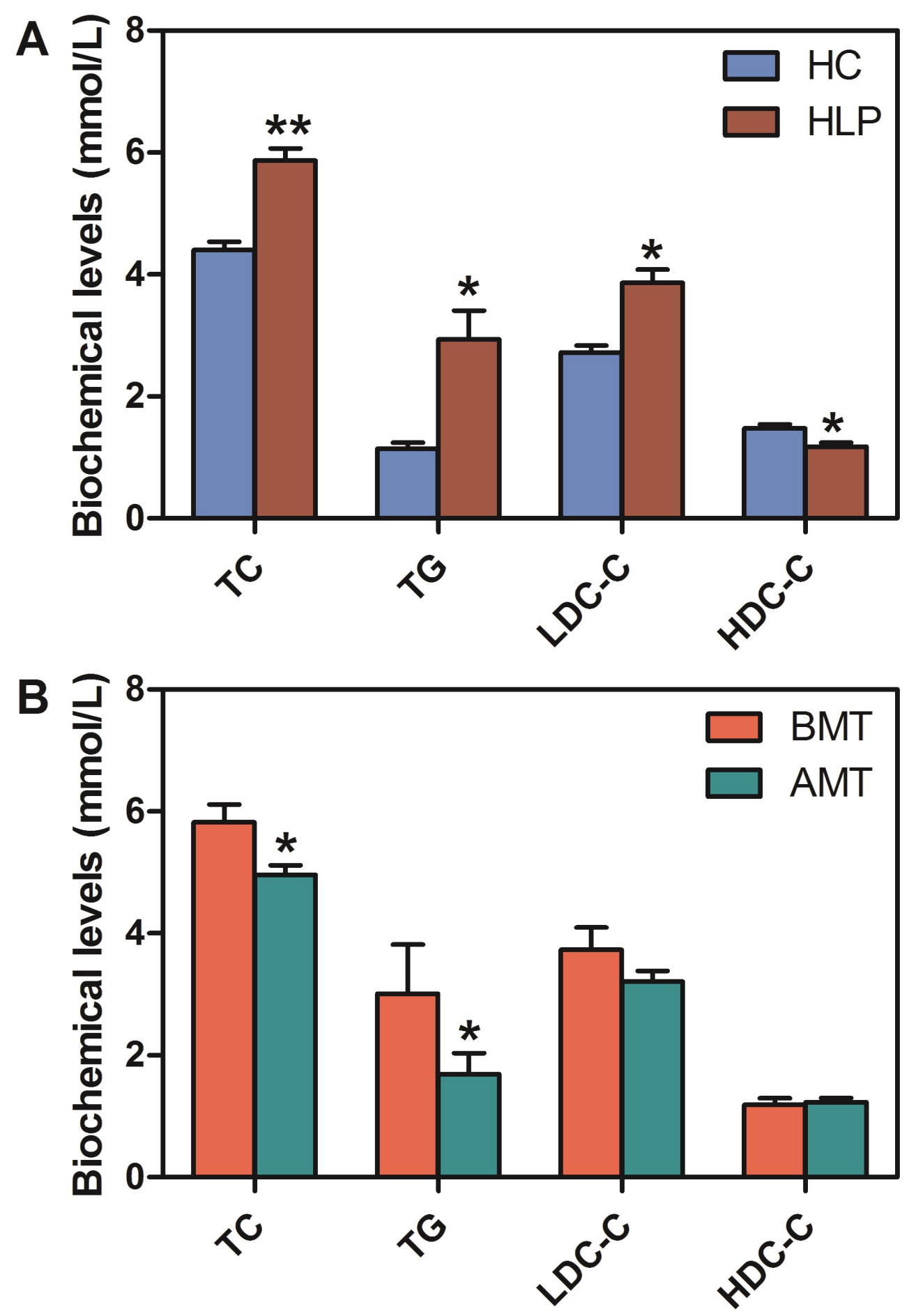

Figure 1. Effects of moxibustion treatment on blood lipid levels in hyperlipidaemia (HLP) patients. A: The levels of total cholesterol (TC), triglyceride (TG), low-density lipoprotein cholesterol (LDC-C) and high-density lipoprotein cholesterol (HDC-C) in 
HLP patients $(n=12)$ and healthy controls $(\mathrm{HC}, \mathrm{n}=23)$; B: The levels of TC, TG, LDC-C and HDC-C in HLP patients with moxibustion treatment (AMT, $n=12$ ) and HLP patients without moxibustion treatment (BMT, $\mathrm{n}=12)$. ${ }^{*} \mathrm{p}$-value $<0.05$; ** p-value $<0.001$.

\subsection{Plasma metabolomics analysis}

Samples comparative analyses were performed using OPLS-DA. The scores plot of OPLS-DA shown that there is a separation between the HLP patients and the HC group (Fig. 2A) and also a separation between AMT group and BMT group (Fig. 2B). All these results implied that the metabolic profiles were different in the two-pair comparison groups. Permutation test for the OPLS-DA model of HLP and HC generated intercepts of $\mathrm{R}^{2}=0.833$ and $\mathrm{Q}^{2}=-0.925$ (Fig. 2C). Permutation test for the OPLS-DA model of AMT and BMT generated intercepts of $\mathrm{R}^{2}=0.479$ and $\mathrm{Q}^{2}=-0.53$ (Fig. 2D). These results implied that the OPLS-DA models had a good predictive ability and were not over-fitted.
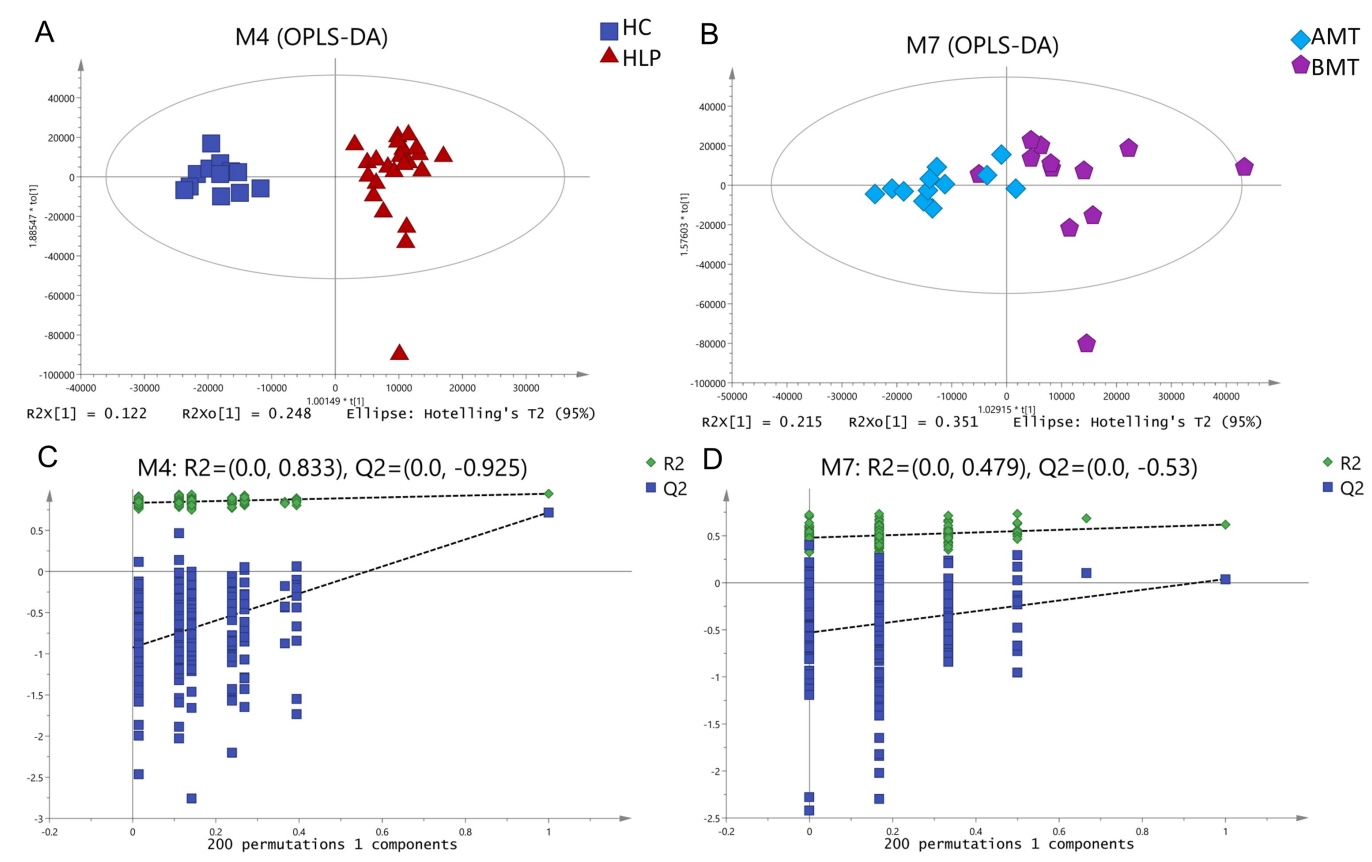

Figure 2 Orthogonal partial lease squares discriminant analysis (OPLS-DA) and its permutation test for plasma samples of HLP, HC, BMT and BMT. A: OPLS-DA score plots between HLP and HC; B: OPLS-DA score plots between BMT and AMT (B); C: permutation test from OPLS-DA models of HLP and HC; D: permutation test from OPLS-DA models of BMT and AMT.

\subsection{Identification of differential metabolites}

Total 12611 metabolites were detected in 47 plasma samples. Compared to HC, 87 metabolites changed (34 metabolites decreased and 53 metabolites increased, Fig. 
3A) markedly in the HLP patients (VIP $>1$, P-value $<0.05$ ). 51 metabolites changed (Fig. 3A, 39 metabolites decreased and 12 metabolites increased) significantly in AMT compared to BMT (VIP $>1$, P-value $<0.05$ ). Among these differential metabolites, 28 metabolites were shared by HLP vs HC and AMT vs BMT (Fig. 3B). 21 out of 28 metabolites had the opposite regulatory relationship (17 metabolites increased in HLP vs HC, but decreased in AMT vs BMT; 4 metabolites decreased in HLP vs HC, but increased in AMT vs BMT).
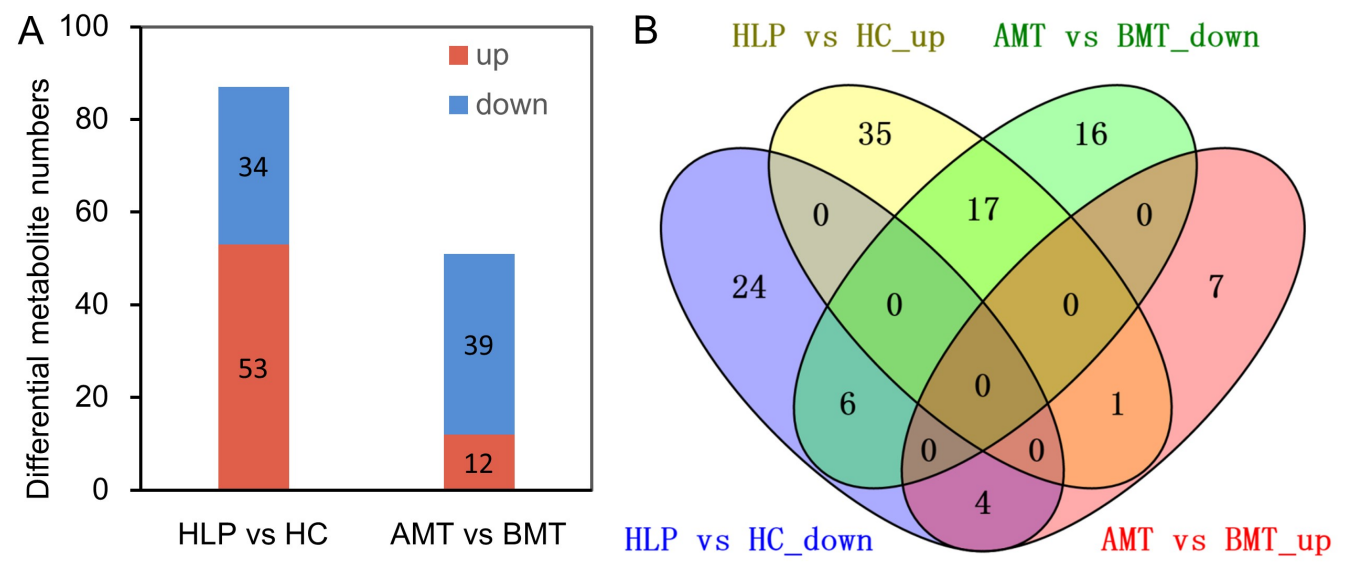

Figure 3 Differential metabolites in HLP vs HC and BMT vs AMT. A: The differential metabolites numbers in HLP vs HC and BMT vs AMT; B: The Venn diagram of differential metabolites shared by HLP vs HC and BMT vs AMT.

\subsection{The comparisons of 21 differential metabolites shared in HLP vs HC and BMT vs AMT}

The metabolites of CL(1'-[22:1(13Z)/22:1(13Z)],3'-[22:1(13Z)/14:1(9Z)])[rac], Coenzyme Q10, DG(O-16:0/18:1(9Z)), Guanosine triphosphate adenosine, PA(18:1(9Z)/22:4(7Z,10Z,13Z,16Z)), PA(O-20:0/22:1(11Z)), PS(18:1(9Z)/22:2(13Z,16Z)), PS(20:0/22:2(13Z,16Z)), PS(20:1(11Z)/22:2(13Z,16Z)), $\mathrm{SM}(\mathrm{d} 18: 0 / 22: 1(13 Z))$, TG(15:0/16:0/20:2(11Z,14Z)),

TG(15:0/16:0/20:3(8Z,11Z,14Z)), TG(16:0/16:0/18:2(9Z,12Z)), TG(16:0/16:0/18:1(9Z)), $\mathrm{TG}(16: 0 / 18: 0 / 18: 2(9 \mathrm{Z}, 12 \mathrm{Z})) \quad$ and $\quad \mathrm{TG}(16: 1(9 \mathrm{Z}) / 16: 1(9 \mathrm{Z}) / 18: 2(9 \mathrm{Z}, 12 \mathrm{Z})) \quad$ were significantly increased in HLP vs HC, but significantly reduced after moxibustion treatment (Fig. 4A). The metabolites of 1-Stearoylglycerophosphoglycerol, PG (16:0/16:0), PS (17:0/22:2(13Z,16Z)) and $\mathrm{SM}(\mathrm{d} 18: 1 / 17: 0)$ were significantly decreased in HLP vs HC, but significantly increased after moxibustion treatment (Fig. 4A). Additionally, FC of differential metabolites in AMT vs BMT were significantly negative with that in HLP vs HC (correlation coefficient $\mathrm{R}=-0.9541$, $\mathrm{p}$-value $<0.001$, Fig. 4B). 


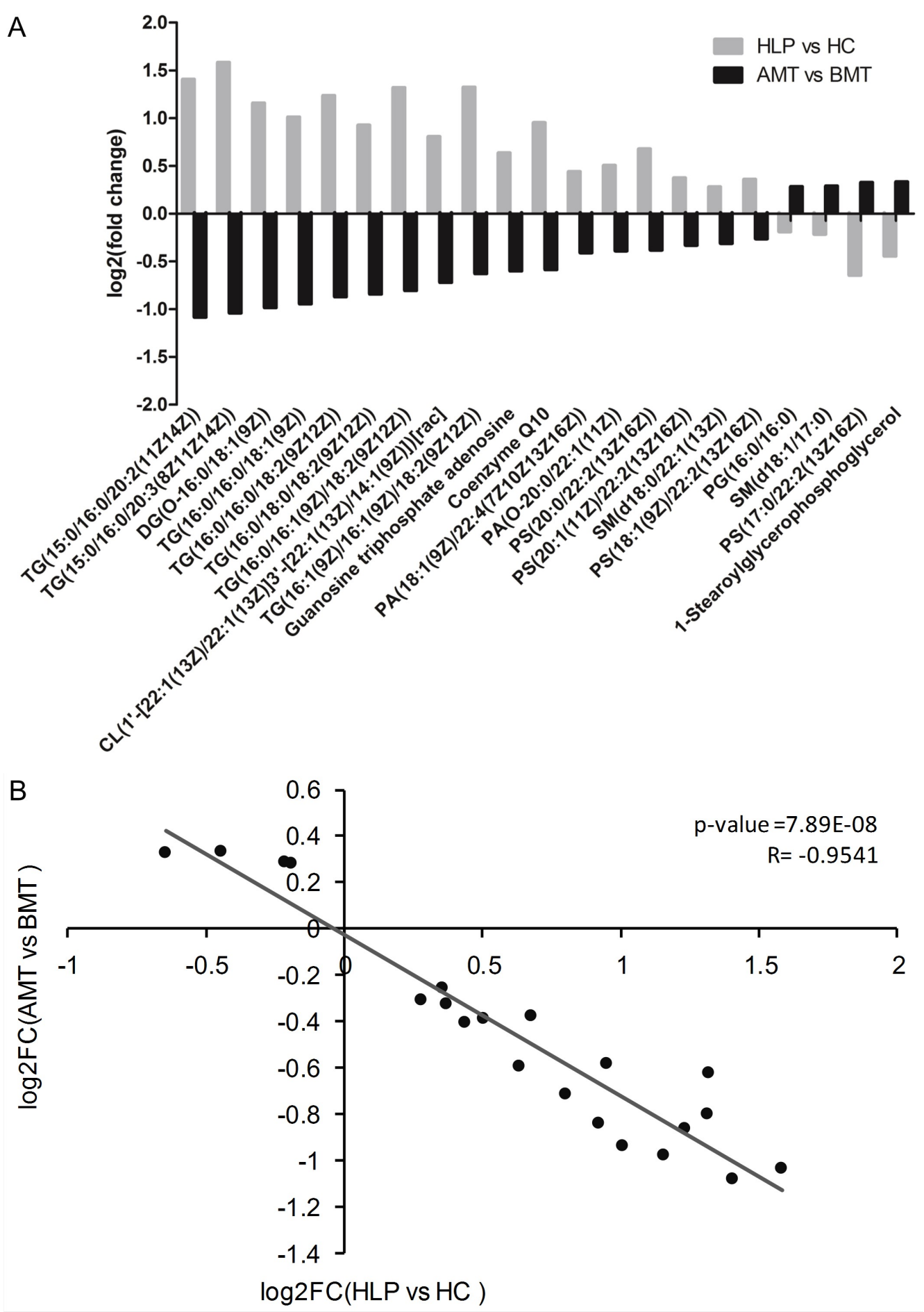

Figure 4 The fold change (FC) of differential metabolites (A) in HLP vs HC and BMT vs AMT and the linear regression analysis of FC data between HLP vs HC and BMT vs AMT (B). Black dots stand for $\log _{2} \mathrm{FC}$ of differential metabolites obtained from HLP vs HC (X-axis) and BMT vs AMT (Y-axis). R: correlation coefficient. 


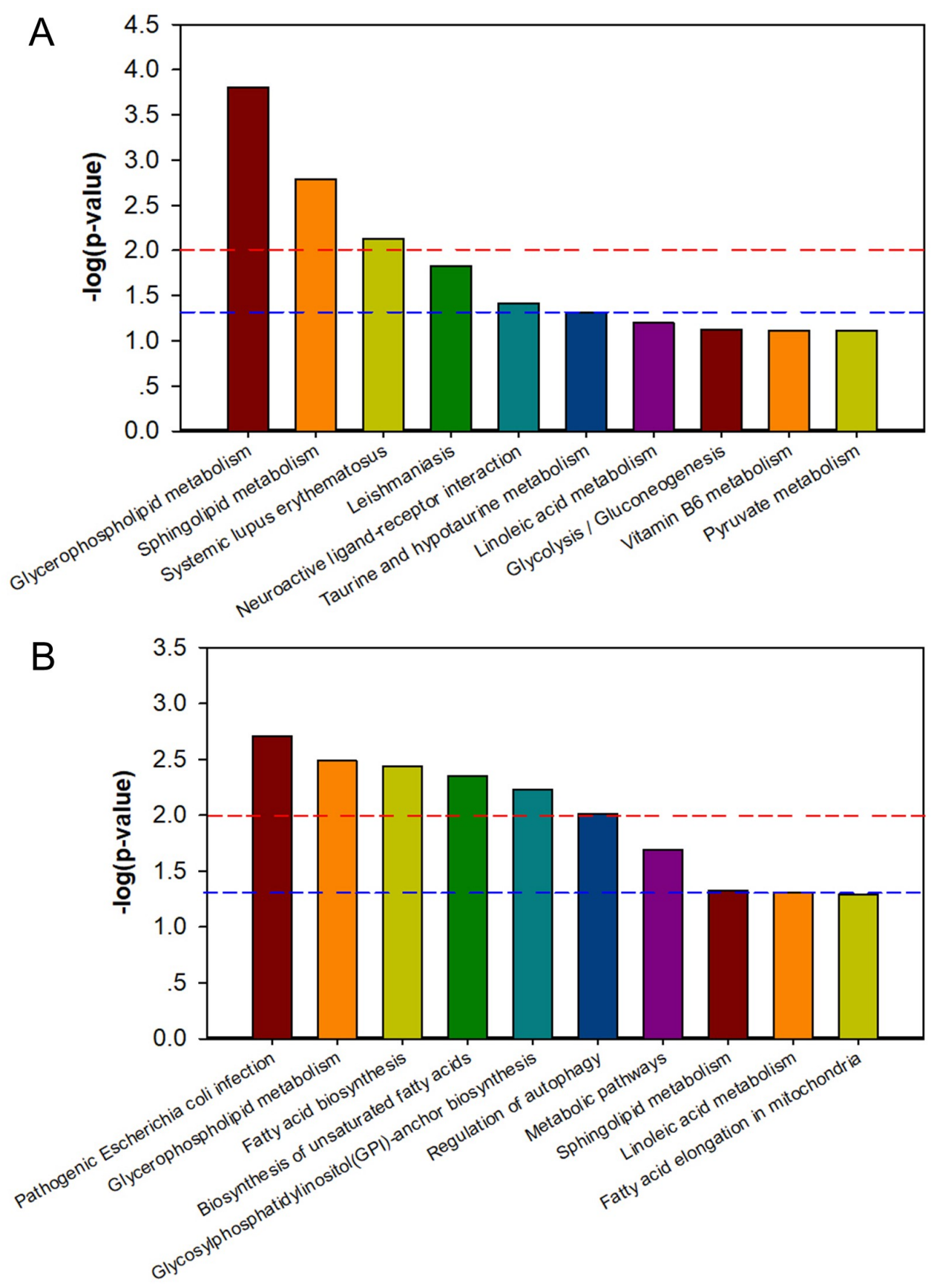

Figure 5 Metabolic pathway analysis of differential metabolites in HLP vs HC (A) and BMT vs AMT (B). The blue and red dotted lines represent $\mathrm{p}$-value $<0.05$ and p-value $<0.01$, respectively.

\subsection{Pathway analysis of differential metabolites}

The top 10 enriched pathways for the differential metabolites of HLP vs HC and AMT vs BMT were shown in Fig. 5A and Fig. 5B, respectively. The differential metabolites in HLP vs HC significantly enriched in the pathways of 
Glycerophospholipid metabolism, SphingolipidSphingolipid metabolism, Systemic lupus erythematosus, Leishmaniasis, Neuroactive ligand-receptor interaction and Taurine and hypotaurine metabolism. The differential metabolites in AMT vs BMT mainly enriched in Pathogenic Escherichia coli infection, Glycerophospholipid metabolism, Fatty acid biosynthesis, Biosynthesis of unsaturated fatty acids, Glycosylphosphatidylinositol (GPI)-anchor biosynthesis, Regulation of autophagy, Metabolic pathways, Sphingolipid metabolism and Linoleic acid metabolism. The pathways of Glycerophospholipid metabolism and Sphingolipid metabolism were shared by the differential metabolites in HLP vs HC and AMT vs BMT. The differential metabolites of AMT vs BMT in the pathways were shown in Table 1. Phosphatidylcholine and phosphatidylethanolamine, which were both decreased after moxibustion treatment, involved in Glycerophospholipid metabolism. A low level of sphingomyelin in the patients with moxibustion treatment was associated with Sphingolipid metabolism.

Table 1. Pathway terms for the differential metabolites in AMT vs BMT

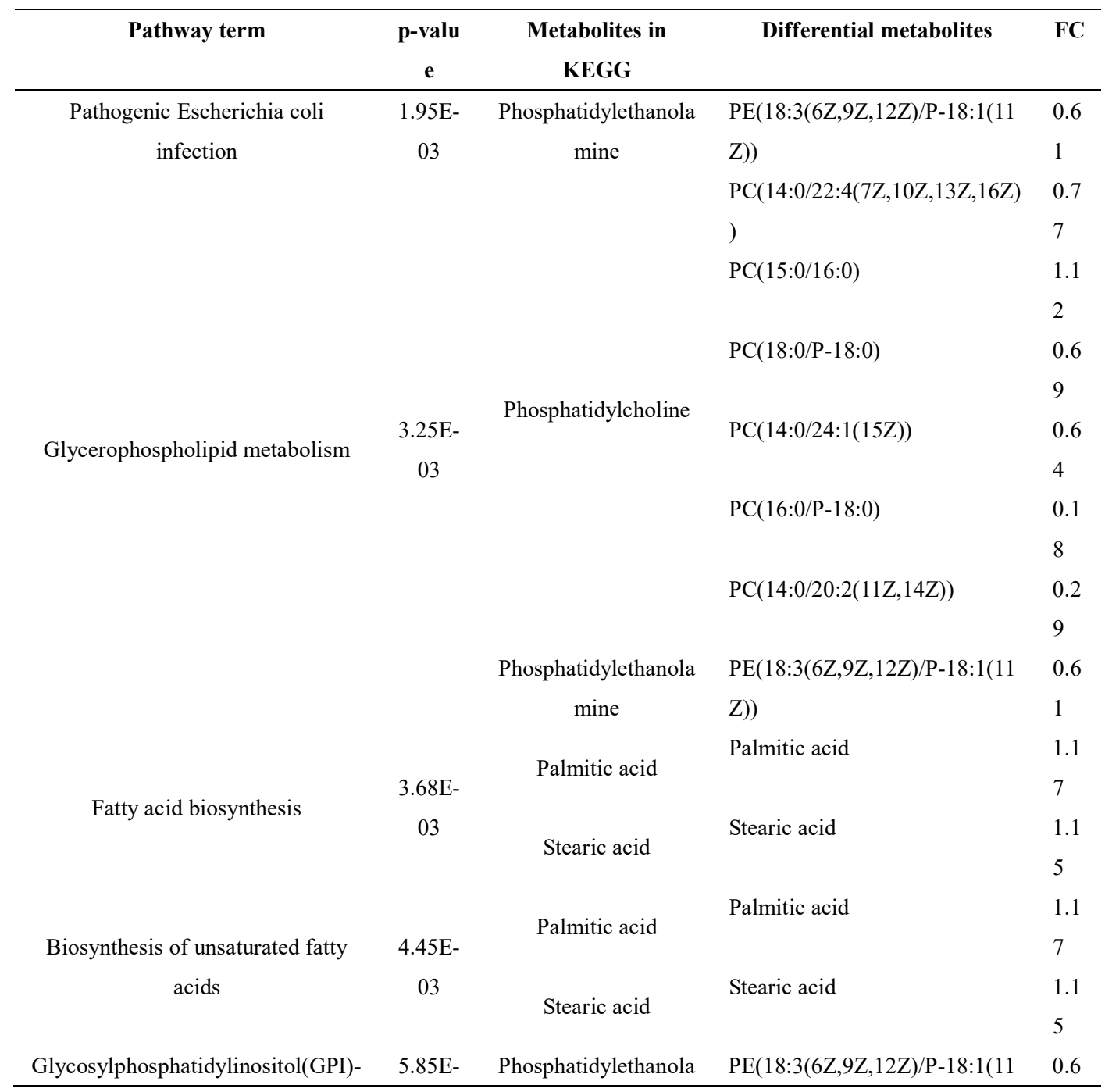




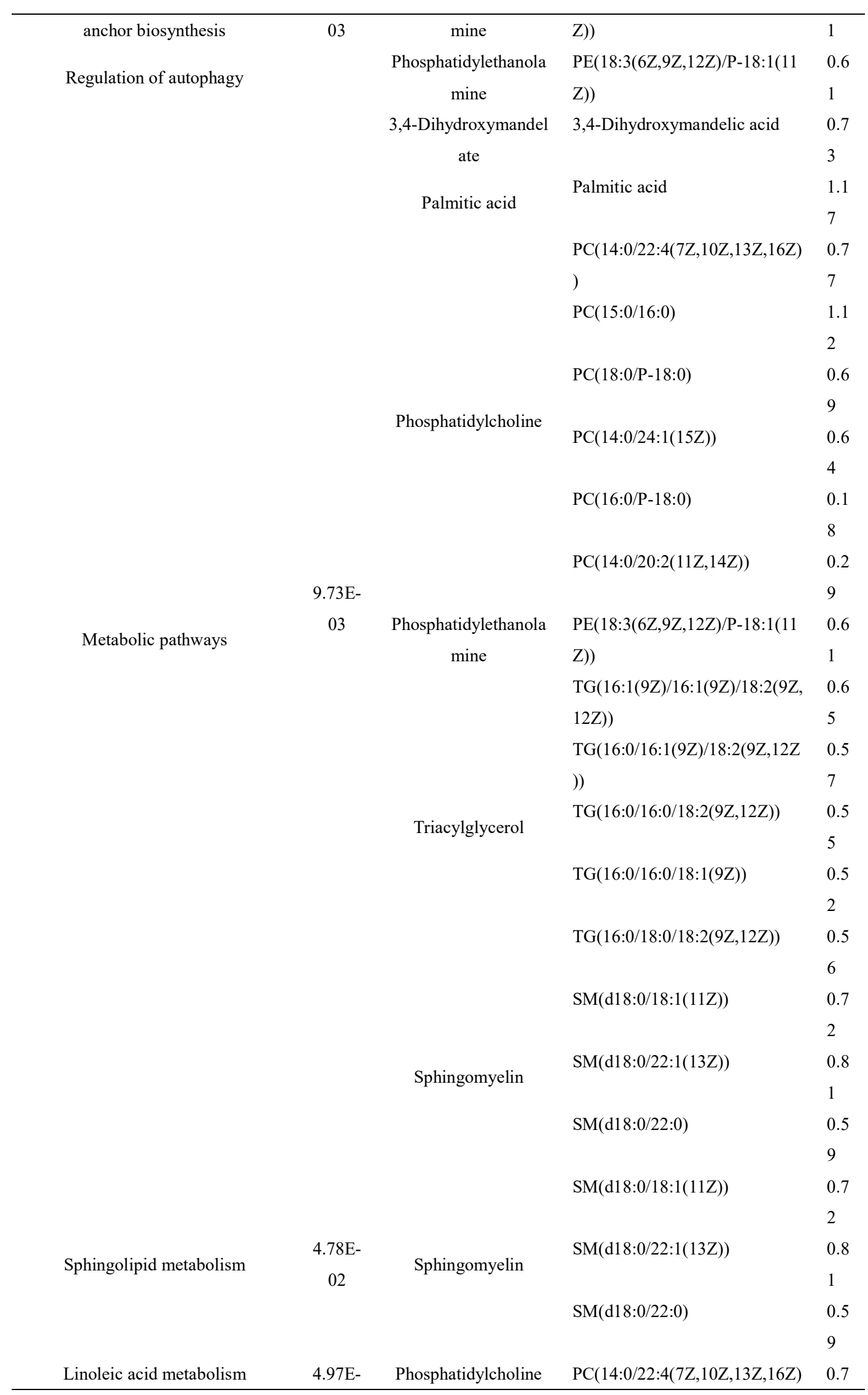




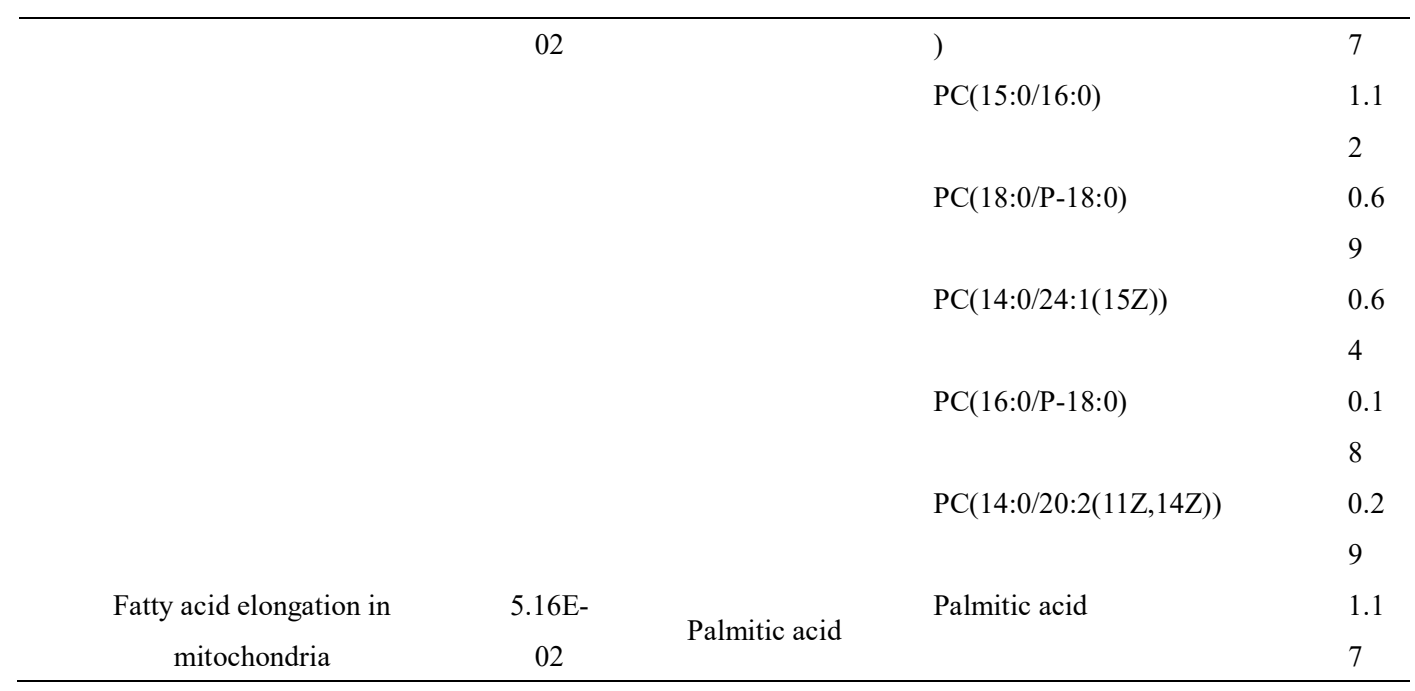

FC: fold change.

\section{Discussion}

Hyperlipidaemia is a widespread disease and a major risk factor for many other diseases. Although lots of chemical drugs, including statins and fibrates, used for the treatment of hyperlipidaemia, most of the drugs are usually expensive and lead to some negative effects, such as cognitive disorder, diabetes mellitus and uterine fibroids [15,16]. Moxibustion and acupuncture had been considered as potential treatments for hyperlipidaemia [8-10]. However, the effect of moxibustion with seed-sized moxa cone on hyperlipidaemia and the metabolic profile of hyperlipidaemia are still unknown. Thus, we did this study based on LC-MS technique.

In this research, high levels of TC, TG and LDL-C along with a low level of HDL-C were detected in the patients with HLP, which were consistent with the literature $[1,17,18]$. Eight-week moxibustion with seed-sized moxa cone treatment significantly down-regulated the levels of TC and TG. There were no significant differences between the AMT group and BMT group in our study. However, the levels of LDC-C and HDC-C in AMT group were both in the normal range. All these results indicated that moxibustion with seed-sized moxa cone had an effective function of anti-hyperlipidaemia.

Based on the LC-MS technique, the plasma metabolite profiles were assessed. Total 87 and 51 differential metabolites were identified in HLP vs HC and AMT vs BMT, respectively. Meanwhile, 21 differential metabolites with opposite alter relation were shared by HLP vs HC and AMT vs BMT and had significantly negative correlations between the two comparisons, implying their important roles in the progression of hyperlipidaemia. The differential metabolites in AMT vs BMT were significantly enriched in lipid metabolism pathways (Glycerophospholipid metabolism, Fatty acid biosynthesis, Biosynthesis of unsaturated fatty acids, 
Sphingolipid metabolism and Linoleic acid metabolism). The pathways of Glycerophospholipid metabolism and Sphingolipid metabolism were shared by the differential metabolites in HLP vs HC and AMT vs BMT. The associated differential metabolites (phosphatidylcholine, phosphatidylethanolamine and sphingomyelin) in the patients with moxibustion treatment might be potential biomarkers.

Recent years, pathways of Glycerophospholipid metabolism and Sphingolipid metabolism had been found to be related to the progression of atherosclerosis, which was accompanied by hyperlipidaemia [19]. The enriched metabolites in pathways of Glycerophospholipid metabolism and Sphingolipid metabolism also have reported that disturbed lipid metabolism plays important roles in the occurrence and development of hyperlipidaemia. The plasma phosphatidylcholine hydroperoxide concentration significantly increased in hyperlipidemia patients and was markedly positively associated with TC and TG levels [20]. Phosphatidylcholine levels were identified as an independent risk factor for cardiovascular disease [21-23]. Soybean phosphatidylcholine prevented lipid accumulation and eased high fat diet-induced hyperlipidaemia via decreasing the levels of $\mathrm{TC}$ and $\mathrm{TG}$ in mice [24]. In the cholesterol-fed rabbits, the concentration and synthetic rate of sphingomyelin increased significantly [25]. The alterations of very low-density lipoproteins composition, which were characterized by a dramatical increase of sphingomyelin, were similar to the lipid composition changes in atherosclerosis [26]. Sphingomyelin content increased in apoE0 mice and promoted the occurrence and development of atherosclerosis [27]. Sphingomyelin inhibition reduced the levels of plasma TC and TG and prevented atherosclerosis in mice [28]. Sphingomyelin levels were determined as an independent risk factor for coronary artery disease [29,30]. In our study, the TC and TG levels were significantly decreased after moxibustion with seed-sized moxa cone treatment. Thus, the moxibustion probably executed its anti-hyperlipidaemia function by lowering the levels of phosphatidylcholine, sphingomyelin, TC and TG.

In conclusion, based on LC-MS technique, we obtained metabolic profiles for HLP, HC, BMT and AMT and identified differential metabolites in the comparisons of HLP vs HC and AMT vs BMT, respectively. High TC and TG levels in HLP patients were reversed by moxibustion with seed-sized moxa cone treatment, indicating its effect of anti-hyperlipidaemia. Moxibustion with seed-sized moxa cone could regulate lipid metabolism pathways, especially Glycerophospholipid metabolism and Sphingolipid metabolism, to against hyperlipidaemia. This is the first research on the treatment effects of moxibustion with seed-sized moxa cone on hyperlipidaemia and the metabolic profile of hyperlipidaemia. The optimal treatment of moxibustion, including moxa dosage, treatment time and treatment cycle, is needed to be studied. The further research of deep molecular mechanisms of hyperlipidaemia is still required.

\section{Materials and methods}




\subsection{Sample collection}

Primary HLP patients $(n=23,10$ males and 13 females) and $\mathrm{HC}(\mathrm{n}=12,4$ males and 8 females) were enrolled in this research. The diagnoses of HLP were according to one or more of the following criteria: (1) TC $>5.20 \mathrm{mmol} / \mathrm{L}$; (2) $\mathrm{TG}>1.7 \mathrm{mmol} / \mathrm{L}$; (3) LDL-C $>3.37 \mathrm{mmol} / \mathrm{L}$ and (4) HDL-C $\leq 1.04 \mathrm{mmol} / \mathrm{L}$. The ages of all participants ranged from 30 to 65 . HLP patients didn't take any lipid-lowering drugs or used lipid-regulating drugs, but had been discontinued for a month. Among the 23 HLP patients, 12 patients were randomly selected to receive moxibustion treatment. The 12 patients were treated by moxibustion with seed-sized moxa cone at "fenglong" of bilateral calves, 7 10 cones at each acupoint, twice a week and continuously for 8 weeks. The blood samples ( $6 \mathrm{ml}$ for each person) were obtained from HLP patients, $\mathrm{HC}$ and HLP patients with moxibustion treatment after 12 hours of fasting. $3 \mathrm{ml}$ blood was used for measurement of biochemical index. Another $3 \mathrm{ml}$ blood was centrifuged at $3000 \mathrm{r} / \mathrm{min}$ at room temperature and stored at $-80^{\circ} \mathrm{C}$. The plasma samples collected before moxibustion treatment and after moxibustion treatment were marked as BMT and AMT, respectively.

All the experimental protocols were approved by the Ethics committee of Jiangsu Province on Integration of Chinese and Western Medicine (the ethical code: 2016LW14) and were performed in accordance with the Declaration of Helsinki. All participants in this research had given a written informed consent.

\subsection{Measurement of biochemical index}

The concentrations of TG, TC, LDC-C and HDC-C were measured by XL-640 automatic analyzer (Erba, Germany).

\subsection{Sample preparation for LC-MS}

Before analysis, $200 \mu \mathrm{l}$ plasma was thawed at room temperature in $1.5 \mathrm{ml}$ Eppendorf tube. $12.5 \mu \mathrm{l}$ cnidium lactonejiae $(100 \mu \mathrm{g} / \mathrm{ml})$ was added as the internal standard. After adding $400 \mu \mathrm{l}$ ice-cold methanol (75\%), the mixture was vortexed for $10 \mathrm{~min}$ and vortexed for another $20 \mathrm{~min}$ together with methyl tertiary butyl ether. Then, after adding $60 \mu \mathrm{l}$ ultrapure water, it was centrifuged at $12500 \mathrm{rpm}$ for $5 \mathrm{~min}$ at $4{ }^{\circ} \mathrm{C} .900 \mu \mathrm{l}$ upper layer of the lipid solution was dried in the SpeedVac evaporating air concentrator. After dissolved with methanol/toluene solvent $(9: 1, \mathrm{v}: \mathrm{v})$, the mixture was vortexed at $17500 \mathrm{rpm}$ for $5 \mathrm{~min}$ at $4{ }^{\circ} \mathrm{C}$. Finally, a $600 \mu \mathrm{l}$ liquid supernatant was harvested for LC-MS analysis.

\subsection{LC-MS conditions}

Liquid chromatography analysis was performed on a $2.6 \mu \mathrm{m}$ column (Kinetex C18). Column temperatures were $55^{\circ} \mathrm{C}$. The condition of the positive ion sample liquid chromatography was as follow: solution A: water/isopropanol (80:20, v:v) 
containing $5 \mathrm{mM}$ ammonium formate and $0.1 \%$ formic acid; solution B: isopropanol /acetonitrile (90:10, v:v) containing $5 \mathrm{mM}$ ammonium formate and $0.1 \%$ formic acid. Gradient elution with a flow rate of $0.3 \mathrm{~mL} / \mathrm{min}$ was used. The elution condition was as follow: $0-4 \mathrm{~min}, 30 \%$ buffer B; $4-5 \mathrm{~min}, 48 \%$ buffer $\mathrm{B}$; $5-22 \mathrm{~min}, 82 \%$ buffer B; 22-23 $\mathrm{min}, 99 \%$ buffer B and $24-35 \mathrm{~min}, 15 \%$ buffer $\mathrm{B}$. The condition of the negative ion sample liquid chromatography was as follow: solution $\mathrm{A}$ : $0.02 \%$ aqueous formate; solution B: acetonitrile. The flow rate and elution condition of gradient elution were the same with that of the positive ion sample.

Mass spectrometry was performed with electrospray ionization in both the positive and negative ion mode. The analytical parameters for mass spectrometry were as follows: ion source temperature: $350^{\circ} \mathrm{C}$; spray voltage: $3.5 \mathrm{KV}$; sheath gas flow velocity: $275 \mathrm{KPa}$; capillary temperature: $350^{\circ} \mathrm{C}$ and auxiliary air velocity: 104 $\mathrm{KPa}$. The full mass scan range was set to $200-1000 \mathrm{~m} / \mathrm{z}$ with the resolution ration of 30000 dpi.

\subsection{Data preprocessing and statistical analysis}

The raw data acquired from LC-MS were analyzed by the progqunesis QI software (Waters Corporation, Milford, USA) with the parameters of precursor tolerance $5 \mathrm{ppm}$, fragment tolerance $10 \mathrm{ppm}$ and retention time (RT) tolerance $0.02 \mathrm{~min}$. The profiles data included $\mathrm{m} / \mathrm{z}$, peak RT and peak intensities; RT-m/z pairs were used as the identifiers for each ion. The combine data of positive and negative data was imported into the SIMCA software package (version 14.0, Umetrics, Umea, Sweden). Supervised orthogonal partial lease squares discriminant analysis (OPLS-DA) was performed to visualize the alterations of metabolites between the groups.

\subsection{Identification of differential metabolites}

Metabolites were identified by the progqunesis QI software (Waters Corporation, Milford, USA) based on Human Metabolome Database (HMDB, http://www.hmdb.ca/), LIPID MAPS database (http://www.lipidmaps.org/) and the self-built database of Shanghai Lu-Ming Biotech Co., Ltd (Shanghai, China). The differential metabolites were screened by the combination of multidimensional analysis and unidimensional analysis. The thresholds were set to variable important for the projection (VIP) obtained from the OPLS-DA $>1$ and $p$-value from a two-tailed Student's test $<0.05$.

\subsection{Pathway analysis for differential metabolites}

In order to identify the effect of disturbed metabolites on metabolic pathways, pathway enrichment analysis for differential metabolites was performed using MBRole 2.0 (http://csbg.cnb.csic.es/mbrole2/) based on Kyoto Encyclopedia of Genes and Genomes (KEGG, http://www.genome.jp/KEGG/pathway.html). The pathway with $\mathrm{p}$-value $<0.05$ was identified as the significant pathway. 


\subsection{Statistical analysis}

All the experiment data of biochemical index were presented as means \pm standard error of mean. Statistical analysis of the experiment data was performed by the software of SPSS 21.0 (IBM Corp Armonk, NY, USA). A two-tailed Student's t-test was used to assess the comparisons between HC group and HLP group and paired t-test were used to assess the contrast between BMT group and AMT group. The significance threshold and extremely significance threshold were set at p-value $<0.05$ $(*)$ and p-value $<0.001(* *)$, respectively.

Author Contributions : Conceptualization, Qinghua Shao and Jie Cheng; Data curation, Guangxia Ni; Formal analysis, Qinghua Shao and Guangxia Ni; Methodology, Jie Cheng and Yi Li; Project administration, Qinghua Shao; Resources, Qinghua Shao, Jie Cheng and Yi Li; Supervision, Qinghua Shao; Validation, Qinghua Shao, Jie Cheng, Yi Li and Guangxia Ni; Visualization, Yi Li; Writing - original draft, Qinghua Shao; Writing - review \& editing, Jie Cheng.

Funding: This work was supported by the science and technique project of Jiangsu Province Bureau of Traditional Chinese Medicine (YB2015048).

Conflict of interest: The authors declare that they have no conflict interest.

\section{Reference}

1. Esteve, E.; Ricart, W.; Fernandez-Real, J.M. Dyslipidemia and inflammation: An evolutionary conserved mechanism. Clin Nutr 2005, 24, 16-31.

2. Merkler, M.; Reiner, Z. The burden of hyperlipidaemia and diabetes in cardiovascular diseases. Fundam Clin Pharmacol 2007, 21 Suppl 2, 1-3.

3. Zhou, X.; Zhang, W.; Liu, X.; Li, Y. Interrelationship between diabetes and periodontitis: Role of hyperlipidemia. Arch Oral Biol 2015, 60, 667-674.

4. Winocour, P.H.; Durrington, P.N.; Ishola, M.; Hillier, V.F.; Anderson, D.C. The prevalence of hyperlipidaemia and related clinical features in insulin-dependent diabetes mellitus. QJ Med 1989, 70, 265-276.

5. Williams, A.D. Hyperlipidaemia and atherogenesis. Med Hypotheses 1990, 33, 213-217.

6. Thompson, G.R. Clinical consequences of hyperlipidaemia. J Inherit Metab Dis 1988, 11 Suppl 1, 18-28.

7. Chou, Y.T.; Chuang, L.P.; Li, H.Y.; Fu, J.Y.; Lin, S.W.; Yang, C.T.; Chen, N.H. Hyperlipidaemia in patients with sleep-related breathing disorders: Prevalence \& risk factors. Indian J Med Res 2010, 131, 121-125. 
8. Xiaorong, C.; Jie, Y.; Zenghui, Y.; Jing, S.; Yaping, L.; Shouxiang, Y.; Xiangping, C. Effects of medicinal cake-separated moxibustion on plasma 6-keto-pgflalpha and txb2 contents in the rabbit of hyperlipemia. $J$ Tradit Chin Med 2005, 25, 145-147.

9. Liu, M.; Hu, W.; Xie, S.; Zhang, J.; Zhao, Z.; Chang, X. [characteristics and laws of acupoint selection in treatment of hyperlipidemia with acupuncture and moxibustion]. Zhongguo Zhen Jiu 2015, 35, 512-516.

10. Liu, M.; Zhang, Q.; Jiang, S.; Zhang, G.; Yue, Z.; Chen, Q.; Zhou, J.; Zou, Y.; Li, D.; Ma, M., et al. Warm-needling acupuncture and medicinal cake-separated moxibustion for hyperlipidemia: Study protocol for a randomized controlled trial. Trials 2017, 18, 310.

11. Xu, H.; Hu, Y.; Gu, Y.; Zhang, H. [effects of moxibustion with seed-sized moxa cone on apoptosis of myocardial cells after sport fatigue in mice]. Zhongguo Zhen Jiu 2015, 35, 257-263.

12. Bai, H.; Lu, S.F.; Chen, W.Y.; Zhong, Z.H.; Gu, Y.H. [effect of moxibustion preconditioning with seed-sized moxa cones on myocardial infarction size and beclin 1 expression in myocardial ischemia-reperfusion injury rats]. Zhen $C i$ Yan Jiu 2017, 42, 471-476.

13. Di, Z.; Jiang, S.; Lin, X.M.; Fu, W.B. [the short-term and long-term effects on neck pain caused by cervical spondylosis treated with combination of acupuncture and moxibustion with seed-sized moxa cone]. Zhongguo Zhen Jiu 2014, 34, 325-328.

14. Liu, Y.; Hou, Z.; Lu, J.; Dong, F.; Wang, P.; Jia, W.; Wang, C. [effects of moxibustion with seed-sized moxa cone at "ganshu" (bl 18) on liver function in rats with precancerous lesion of hepatic cellular cancer]. Zhongguo Zhen Jiu 2015, 35, 702-706.

15. Sattar, N.; Preiss, D.; Murray, H.M.; Welsh, P.; Buckley, B.M.; de Craen, A.J.; Seshasai, S.R.; McMurray, J.J.; Freeman, D.J.; Jukema, J.W., et al. Statins and risk of incident diabetes: A collaborative meta-analysis of randomised statin trials. Lancet 2010, 375, 735-742.

16. Borahay, M.A.; Fang, X.; Baillargeon, J.G.; Kilic, G.S.; Boehning, D.F.; Kuo, Y.F. Statin use and uterine fibroid risk in hyperlipidemia patients: A nested case-control study. Am J Obstet Gynecol 2016, 215, 750 e 751-750 e758.

17. Wasan, K.M.; Pritchard, P.H.; Ramaswamy, M.; Wong, W.; Donnachie, E.M.; Brunner, L.J. Differences in lipoprotein lipid concentration and composition modify the plasma distribution of cyclosporine. Pharm Res 1997, 14, 
1613-1620.

18. Hu, C.; Kong, H.; Qu, F.; Li, Y.; Yu, Z.; Gao, P.; Peng, S.; Xu, G. Application of plasma lipidomics in studying the response of patients with essential hypertension to antihypertensive drug therapy. Mol Biosyst 2011, 7, 3271-3279.

19. Dang, V.T.; Huang, A.; Zhong, L.H.; Shi, Y.; Werstuck, G.H. Comprehensive plasma metabolomic analyses of atherosclerotic progression reveal alterations in glycerophospholipid and sphingolipid metabolism in apolipoprotein e-deficient mice. Sci Rep 2016, 6, 35037.

20. Kinoshita, M.; Oikawa, S.; Hayasaka, K.; Sekikawa, A.; Nagashima, T.; Toyota, T.; Miyazawa, T. Age-related increases in plasma phosphatidylcholine hydroperoxide concentrations in control subjects and patients with hyperlipidemia. Clin Chem 2000, 46, 822-828.

21. Wang, Z.; Klipfell, E.; Bennett, B.J.; Koeth, R.; Levison, B.S.; Dugar, B.; Feldstein, A.E.; Britt, E.B.; Fu, X.; Chung, Y.M., et al. Gut flora metabolism of phosphatidylcholine promotes cardiovascular disease. Nature 2011, 472, 57-63.

22. Tang, W.H.; Wang, Z.; Levison, B.S.; Koeth, R.A.; Britt, E.B.; Fu, X.; Wu, Y.; Hazen, S.L. Intestinal microbial metabolism of phosphatidylcholine and cardiovascular risk. N Engl J Med 2013, 368, 1575-1584.

23. Ierardi, E.; Sorrentino, C.; Principi, M.; Giorgio, F.; Losurdo, G.; Di Leo, A. Intestinal microbial metabolism of phosphatidylcholine: A novel insight in the cardiovascular risk scenario. Hepatobiliary Surg Nutr 2015, 4, 289-292.

24. Lee, H.S.; Nam, Y.; Chung, Y.H.; Kim, H.R.; Park, E.S.; Chung, S.J.; Kim, J.H.; Sohn, U.D.; Kim, H.C.; Oh, K.W., et al. Beneficial effects of phosphatidylcholine on high-fat diet-induced obesity, hyperlipidemia and fatty liver in mice. Life Sci 2014, 118, 7-14.

25. McCandless, E.L.; Zilversmit, D.B. The effect of cholesterol on the turnover of lecithin, cephalin and sphingomyelin in the rabbit. Arch Biochem Biophys 1956, 62, 402-410.

26. Rodriguez, J.L.; Ghiselli, G.C.; Torreggiani, D.; Sirtori, C.R. Very low density lipoproteins in normal and cholesterol-fed rabbits: Lipid and protein composition and metabolism. Part 1. Chemical composition of very low density lipoproteins in rabbits. Atherosclerosis 1976, 23, 73-83.

27. Jeong, T.; Schissel, S.L.; Tabas, I.; Pownall, H.J.; Tall, A.R.; Jiang, X. 
Increased sphingomyelin content of plasma lipoproteins in apolipoprotein e knockout mice reflects combined production and catabolic defects and enhances reactivity with mammalian sphingomyelinase. $J$ Clin Invest 1998, 101, 905-912.

28. Park, T.S.; Panek, R.L.; Mueller, S.B.; Hanselman, J.C.; Rosebury, W.S.; Robertson, A.W.; Kindt, E.K.; Homan, R.; Karathanasis, S.K.; Rekhter, M.D. Inhibition of sphingomyelin synthesis reduces atherogenesis in apolipoprotein e-knockout mice. Circulation 2004, 110, 3465-3471.

29. Jiang, X.C.; Paultre, F.; Pearson, T.A.; Reed, R.G.; Francis, C.K.; Lin, M.; Berglund, L.; Tall, A.R. Plasma sphingomyelin level as a risk factor for coronary artery disease. Arterioscler Thromb Vasc Biol 2000, 20, 2614-2618.

30. Schlitt, A.; Blankenberg, S.; Yan, D.; von Gizycki, H.; Buerke, M.; Werdan, K.; Bickel, C.; Lackner, K.J.; Meyer, J.; Rupprecht, H.J., et al. Further evaluation of plasma sphingomyelin levels as a risk factor for coronary artery disease. Nutr Metab (Lond) 2006, 3, 5. 